\title{
Evaluation of Weight Reduction, Diabetic State and Satiety Hormones After Laparoscopic Greater Curvature Plication in Morbidly Obese Diabetic Patients
}

\author{
Waleed Omar, Mohammad Hamed*, Sabry Ahmed Mahmoud, Hosam Elbanna, \\ Mahmoud Abdelnaby, Sameh Emile, Mohamed Anwar \\ General Surgery Department, Mansoura University Hospitals, Mansoura University, Mansoura, Egypt \\ Email address: \\ waleed_h_omar@yahoo.com (W. Omar), mohammadhamed6789@gmail.com (M. Hamed), Sabrybadr@hotmail.com (S. A. Mahmoud), \\ hosamelbanna@hotmail.com (H. Elbanna), abdelshafymahmoud@gmail.com (M. Abdelnaby), sameh200@hotmail.com (S. Emile), \\ drmohamedanwar1981@yahoo.com (M. Anwar) \\ ${ }^{*}$ Corresponding author
}

\section{To cite this article:}

Waleed Omar, Mohammad Hamed, Sabry Ahmed Mahmoud, Hosam Elbanna, Mahmoud Abdelnaby, Sameh Emile, Mohamed Anwar. Evaluation of Weight Reduction, Diabetic State and Satiety Hormones After Laparoscopic Greater Curvature Plication in Morbidly Obese Diabetic Patients. Journal of Surgery. Vol. 6, No. 2, 2018, pp. 43-49. doi: 10.11648/j.js.20180602.13

Received: February 6, 2018; Accepted: February 24, 2018; Published: March 19, 2018

\begin{abstract}
Bariatric surgery proved effective in the treatment of morbid obesity and its associated comorbidities. The aim of this study was to assess weight loss and changes in glucose homeostasis and satiety hormones and to evaluate improvement in diabetic status in morbidly obese patients with type two diabetes mellitus (T2DM) at 6 and 12 months after laparoscopic greater curvature plication (LGCP). Twenty patients with morbid obesity with T2DM were operated upon with LGCP. Weight loss was assessed by the decrease in BMI and percentage of excess weight loss at 6 and 12 months after LGCP. Fasting and postprandial blood glucose levels, HbA1c, fasting serum insulin, serum ghrelin and glucagon-like peptide 1 (GLP-1) levels were measured before and at 6 and 12 months postoperatively. The mean age of patients was 37.6 years. There was significant decrease in BMI from 45.4 to 40.1 at 6 months $(p=0.0008)$ and then to 36.4 at 12 month $(p<0.0001)$. The mean fasting blood glucose decreased significantly from 134.8 preoperatively to 120.8 at 6 months $(\mathrm{p}<0.0001)$ and then to 109.5 at 12 months $(\mathrm{p}<$ 0.0001). The mean preoperative HbAlc declined from 6.8 before LGCO to 6.3 at 6 months $(\mathrm{p}<0.0001)$ then to 5.9 at 12 months ( $p<0.0001)$. The mean fasting insulin level decreased from 20.5 preoperatively to 17.4 at 6 months $(p<0.0001)$ then to 16.7 at 12 months $(\mathrm{p}<0.0001)$. The mean baseline ghrelin level decreased significantly from 551.7 preoperatively to 441.5 at 6 months $(p<0.0001)$ then to 422.5 at 12 months $(p<0.0001)$. The mean GLP-1 declined from 33.7 before surgery to 33.5 at 6 months $(p=0.76)$ then to 33.1 at 12 months $(p=0.36)$. LGCP is an effective bariatric procedure that achieved satisfactory weight loss and significant improvement in the glycemic control as demonstrated by improvement in laboratory markers as serum glucose, insulin, and ghrelin hormone levels.
\end{abstract}

Keywords: Laparoscopic, Greater Curvature, Plication, Diabetes, Obesity

\section{Introduction}

Obesity is a major worldwide health problem, since 1997 the World Health Organization has recognized it as a global epidemic and in 2005 more than 400 million obese adults were recorded [1]. While conservative measures as dieting and physical exercise have proven to be inadequate, bariatric surgery proved to be an effective alternative and has become very popular in the last decade [2]. Common techniques of bariatric surgery aim to reduce gastric capacity, cause malabsorption, or both [3].

Obesity plays a vital role in the pathogenesis of insulin resistance and type 2 diabetes mellitus (T2DM). Morbidly obese patients are at higher risk of T2DM, hypertension, hyperlipidemia, and cardiovascular disease. Bariatric surgery is currently the most effective treatment for weight loss and is also shown to lead to rapid and prolonged improvement in insulin resistance and hyperglycemia in the majority of 
morbidly obese T2DM patients [4].

Gatstric plication is a restrictive bariatric technique that was first reported by Wilkinson in 1981 [5]. Laparoscopic greater curvature plication (LGCP) successfully reduces the gastric volume by plication of the greater curvature without the use of foreign materials or resection of a part of the stomach. It has been thought that a lack of stomach resection will reduce the postoperative risks of gastric leakage [6].

Current reports have shown that LGCP results in effective weight loss in the short and medium term [7]. In a recent study, most of the patients with T2DM exhibited significant improvement or resolution of their diabetic status at 6 months after the procedure [8]. Furthermore, it has been shown that LGCP is a safe procedure as low complication rates have been reported so far in the short and medium-term, however more evidence is required in order to establish the long-term efficacy and safety of LGCP [9].

To date, there is lack of studies focusing on the effects of LGCP on the glycemic control in patients with T2DM. Particularly, there are no reports regarding the metabolic and hormonal changes that are associated with improvement in T2DM following LGCP.

A recent study [10] found the improvement in the glycemic control after LGCP to be inferior when compared with laparoscopic sleeve gastrectomy (LSG) even if the complications are lower. The authors reported a decrease in mean $\mathrm{HbA} 1 \mathrm{c}$ after LGCP from $7.9 \%$ preoperatively to $7.5 \%$ postoperatively, and no patients stopped their anti-diabetes medications despite the fact that all patients were on oral hypoglycemic medications and the history of their diabetes did not exceed 5 years. This may indicate that LGCP has a weaker metabolic effect compared with other restrictive procedures..

The aim of this prospective study was to assess weight loss and explore changes in glucose homeostasis and satiety hormones and to evaluate improvement in diabetic status in morbidly obese patients with T2DM at 6 and 12 months after LGCP.

\section{Patients and Methods}

\subsection{Study Design and Setting}

This is a prospective cohort study on morbidly obese patients with type 2 DM who were admitted to general surgery department of Mansoura University Hospitals during the period of May 2015 to December 2016 were submitted to LGCP were included to the study Ethical approval for the study was obtained from the Institutional Review Board (IRB) of Mansoura Faculty of Medicine.

\subsection{Inclusion Criteria}

Adult patients of both genders with morbid obesity (BMI > $35 \mathrm{~kg} / \mathrm{m} 2$ ) with type $2 \mathrm{DM}$ were included. Patients with other associated co-morbidities as hypertension and hypertriglyceridemia were also included to the study.

\subsection{Exclusion Criteria}

We excluded elderly patients aging more than 60 years, patients with secondary obesity due to endocrine disorders, patient unfit for general anesthesia, patients who had not attempted non-surgical weight loss treatment in the past, patients with psychological conditions that influence his/her perception of the study protocol, and patients with previous surgery for morbid obesity.

Thorough history was taken from all patients regarding the duration of obesity, associated comorbidities, upper GI symptoms including GERD and dyspepsia, current medications, and previous treatments received for obesity. The weight and height of patients were inquired and recorded. Dietary history was taken with emphasis on the degree of appetite, type and number of meals and snacks in between meals, frequency of drinking water and beverages, and any food allergies. Patients were asked about the symptoms and duration of type $2 \mathrm{DM}$, results of investigations, treatment received whether in the form of oral anti-diabetic medications or insulin, and complications of DM.

Physical examination was conducted to exclude secondary obesity especially to endocrine disorders, and to exclude presence of abdominal masses or hernias. Anthropometric measurements were performed for all patients, body weight was measured to the nearest $0.5 \mathrm{~kg}$ and height to the nearest $1 \mathrm{~cm}$. BMI was calculated as body weight in kilograms divided by the square of the height in meters.

All patients were evaluated with routine laboratory investigations in the form of complete blood count, serum albumin, serum bilirubin, AST and ALT enzymes, serum creatinine, and prothrombin time. Assessment of the diabetic condition was done by measurement of fasting and 2-hour postprandial serum glucose levels, HbAlc, and plasma insulin levels. Venous levels of satiety hormones including Ghrelin and Glucagon like peptide -1 (GLP-1) were measured after an overnight fast.

Upper GI endoscopy, barium study and abdominal ultrasound were performed to evaluate the condition of the stomach and exclude associated pathologies as gallstones.

\subsection{Surgical Technique}

Prophylactic measures against thrombo-embolism were taken and proton pump inhibitors and third generation cephalosporin were administered with the induction of anesthesia.

Surgical procedures were done under general anesthesia, in the supine position with30-degree reverse trendelenburg. Veress needle was inserted just above the umbilicus with adistance above umbilicus and pneumoperitoneum was created by $\mathrm{CO} 2$ insufflation at $15 \mathrm{mmHg}$ pressure via. The first trocar was placed to the left of midline above the umbilicus depending on body mass. This trocar was used for introduction of the telescope (telescope trocar). Two trocars were inserted at the mid-clavicular line to the right and to the left of the first trocar. The fourth trocar was placed at the left 
mid-axillary line just below the subcostal line. The fifth trocar was inserted at the midline $4 \mathrm{~cm}$ below the xyphoid process for introduction of liver retractor.

After opening the lesser sac at the junction between the body and pylorus, the devascularization of the greater curvature started by grasping and upward pulling of the anterior gastric wall at the prepyloric region then the greater omentum was dissected close to the gastric wall starting from prepyloric area up to $2 \mathrm{~cm}$ to the angle of Hiss preserving the anatomy of the angle of Hiss and sacrificing left and right gastroepiploic vessels. Devasculaiztion of the greater curvature was done using LigaSure ${ }^{\mathrm{TM}}$ or Ultracision Harmonic Scalpelas shown in figure 1.

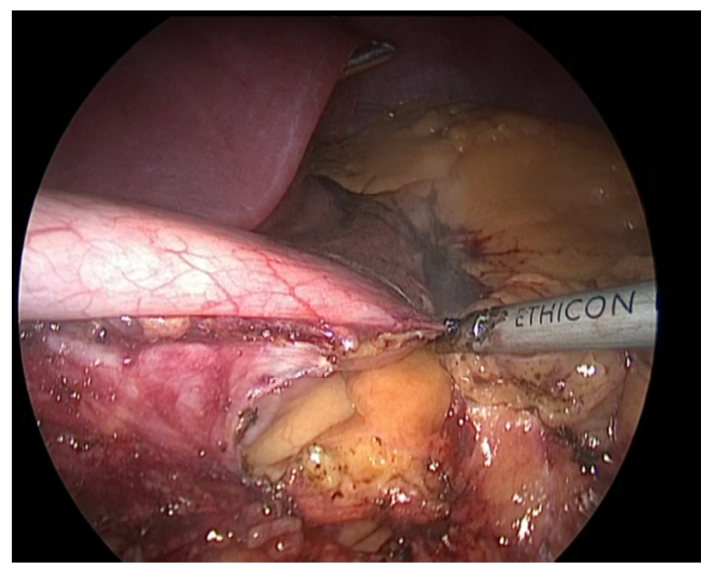

Figure 1. Devascularization of greater curvature.

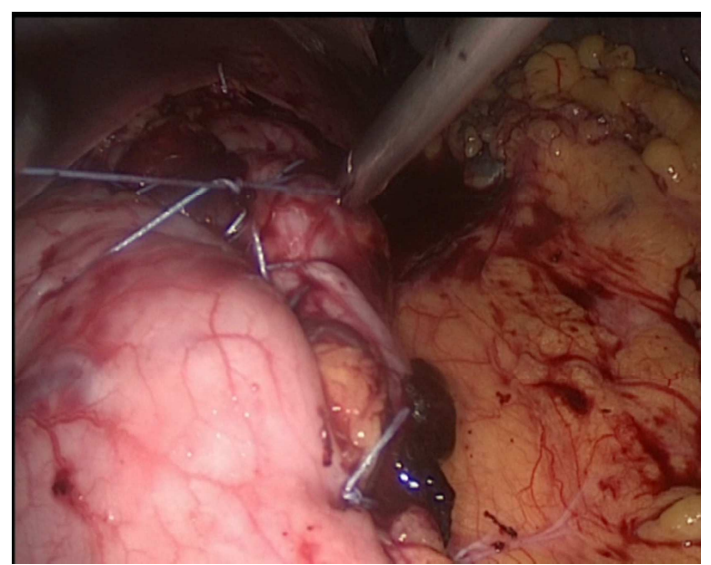

Figure 2. Plication of the greater curvature.

Plication of the greater curvature of the stomach was done using non-absorbable polyfilament sutures (Ethibond $2 / 0$ or Ticron 2/0) with round needle. Invagination of the greature curvature of the stomach was performed by two-row plication technique.

A No. $36 \mathrm{r}$ boogie was introduced transorally into the stomach. The first row composed of interrupted fullthickness sutures. Deep bites of the posterior then the anterior gastric wall were taken $4 \mathrm{~cm}$ away from the greater curvature starting $2 \mathrm{~cm}$ away from the angle of His and ending at the prepyloric region.

The second row of plication composed of continuous sero- muscular sutures starting $2 \mathrm{~cm}$ away from the angle of His and ending at the prepyloric region, the second row was placed $1 \mathrm{~cm}$ away from the first plication suture line as shown in figure 2.

Patients were monitored postoperatively in terms of vital signs, Resumption of oral feeding was scheduled and patients start drinking oral fluids once the patient recovered after surgery.

Patients were discharged after 48 hours of operation. Proton pump inhibitors (pantoprazole, $40 \mathrm{mg}$ ) were prescribed for 3 weeks postoperatively. After discharge, liquid diet was prescribed for each patient for 2 weeks, pureed food for 2 weeks, and then solid food gradually.

\subsection{Outcomes of the Study}

The primary outcome of the study was improvement in T2DM after LGCP as shown by decrease in fasting and postprandial blood glucose levels, $\mathrm{HbAlc}$, fasting serum insulin, and reduction of the dose of oral anti-diabetic medications. Secondary outcomes include weight loss as demonstrated by decrease in BMI and percentage of excess weight loss, and changes in serum ghrelin and glucagon-like peptide 1 (GLP-1) levels after LGCP.

\subsection{Follow up}

Patients were instructed to visit the outpatient clinic at one week for suture removal then at 6 and 12 months postoperatively. During follow-up visits patients were assessed with regards weight loss, improvement in comorbidities, plasma glucose and insulin levels, HbA1c levels, and satiety hormones (Ghrelin). Patients were asked if they reduced or stopped taking anti-diabetic medications.

\subsection{Statistical Analysis}

The data were collected and entered into the personal computer. Statistical analysis was done using Statistical Package for Social Sciences (SPSS/version 15) software. Continuous data were expressed as mean and standard deviation (SD) or median and range and were analyzed using student t-test. Categorical data were expressed as number and percentage and were analyzed using Fisher's exact test. $\mathrm{P}<0.05$ was considered statistically significant.

\section{Results}

Twenty diabetic patients with morbid obesity underwent laparoscopic greater curvature plication and were enrolled in this study. Patients were 19 (95\%) females and one (5\%) male. The mean age of patients was $37.6 \pm 8.7$ years ranging from 22 to 52 years.

The mean height of the patients was $158.1 \pm 5.3 \mathrm{~cm}$, ranging from 150 to $171 \mathrm{~cm}$, the mean preoperative weight was $113 \pm 12.8$ (range, 95- 146) kilograms. The mean baseline body mass index (BMI) was $45.4 \pm 4.05$ (41- 53.7) $\mathrm{Kcal} / \mathrm{m}^{2}$. Three patients had arterial hypertension and two patients complained of joint pain. 
Regarding the diabetic status, the mean preoperative HbA1c was $6.8 \pm 0.24$ (range, 6.1- 7.3) \%, the mean fasting blood glucose was $134.8 \pm 10.8$ (range, $117-152) \mathrm{mg} / \mathrm{dL}$, and the mean fasting insulin level was $20.5 \pm 2.1$ (range, 18-24) $\mathrm{mIU} / \mathrm{L}$.

The mean baseline ghrelin level was $551.7 \pm 17.1$ (range, 522- 576) $\mathrm{pg} / \mathrm{mL}$, and the mean GLP-1 was $33.7 \pm 2.3$ (range, 30- 38) $\mathrm{pg} / \mathrm{mL}$.

The mean operative time was $76.5 \pm 5.4$ (range, 60-90) minutes. There was no conversion to open surgery. Minor complications were recorded in six patients in the form of postoperative vomiting up to four weeks after surgery. There was no mortality in the study. The median hospital stay was 2 days (range, 1-3 days).

Regarding weight loss, there was significant decrease in body weight from $113 \pm 12.8$ preoperatively to $100.5 \pm 15.3$ at 6 months $(\mathrm{p}=0.007)$ and then to $91.1 \pm 13.7$ at 12 months $(\mathrm{p}<$ $0.0001)$. Similarly, the mean BMI declined significantly from $45.4 \pm 4.05$ to $40.1 \pm 5.1$ at 6 months $(p=0.0008)$ and then to $36.4 \pm 4.7$ at 12 month $(\mathrm{p}<0.0001)$. Table 1

Table 1. Weight loss after laparoscopic gastric plication.

\begin{tabular}{|c|c|c|c|c|c|}
\hline Variable & Preoperative & Postoperative at 6 months & $P$ value & Postoperative at 12 months & $P$ value \\
\hline Body weight & $113 \pm 12.8$ & $100.5 \pm 15.3$ & 0.007 & $91.1 \pm 13.7$ & $<0.0001$ \\
\hline BMI & $45.4 \pm 4.05$ & $40.1 \pm 5.1$ & 0.0008 & $36.4 \pm 4.7$ & $<0.0001$ \\
\hline EWL (\%) & ----- & $24.6 \pm 8.4$ & ---- & $41.4 \pm 12.7$ & $<0.0001$ \\
\hline
\end{tabular}

Regarding the improvement in diabetic status, 18 (90\%) patients showed significant improvement in DM, four had complete resolution and stopped taking anti-diabetic medications and 14 required lower dose of anti-diabetic medications.

The mean fasting blood glucose decreased significantly from $134.8 \pm 10.8$ preoperatively to $120.8 \pm 7.8$ at 6 months $(\mathrm{p}<0.0001)$ and then to $109.5 \pm 7.9$ at 12 months $(\mathrm{p}<0.0001)$. The mean postprandial blood glucose decreased significantly from $183.8 \pm 21.2$ preoperatively to $147.8 \pm 12.02$ at six months $(\mathrm{p}<0.0001)$ then to $138.6 \pm 1.4$ at 12 months $(\mathrm{p}<$ $0.0001)$.

The mean preoperative HbAlc declined from $6.8 \pm 0.24$ before gastric plication to $6.3 \pm 0.22$ at 6 months $(p<0.0001)$ then to $5.9 \pm 0.24$ at 12 months $(p<0.0001)$. The mean fasting insulin level decreased from $20.5 \pm 2.1$ preoperatively to $17.4 \pm 1.7$ at 6 months $(\mathrm{p}<0.0001)$ then to $16.7 \pm 1.6$ at 12 months $(\mathrm{p}<0.0001)$ as shown in table 2 .

Table 2. Improvement in diabetic status after laparoscopic gastric plication.

\begin{tabular}{|c|c|c|c|c|c|}
\hline Variable & Preoperative & Postoperative at 6 months & $P$ value & Postoperative at 12 months & $P$ value \\
\hline Fasting blood glucose & $134.8 \pm 10.8$ & $120.8 \pm 7.8$ & $<0.0001$ & $109.5 \pm 7.9$ & $<0.0001$ \\
\hline Postprandial blood glucose & $183.8 \pm 21.2$ & $147.8 \pm 12.02$ & $<0.0001$ & $138.6 \pm 1.4$ & $<0.0001$ \\
\hline HbAlc & $6.8 \pm 0.24$ & $6.3 \pm 0.22$ & $<0.0001$ & $5.9 \pm 0.24$ & $<0.0001$ \\
\hline Fasting insulin level & $20.5 \pm 2.1$ & $17.4 \pm 1.7$ & $<0.0001$ & $16.7 \pm 1.6$ & $<0.0001$ \\
\hline
\end{tabular}

Regarding changes in GI hormones, the mean baseline ghrelin level decreased significantly from $551.7 \pm 17.1$ preoperatively to $441.5 \pm 21.5$ at 6 months $(\mathrm{p}<0.0001)$ then to $422.5 \pm 16.8$ at 12 months $(\mathrm{p}<0.0001)$. The mean
GLP-1 declined from $33.7 \pm 2.3$ before surgery to $33.5 \pm$ 1.8 at 6 months $(p=0.76)$ then to $33.1 \pm 1.8$ at 12 months $(\mathrm{p}=0.36)$, however this decline was not significant as shown in table 3 .

Table 3. Changes in gastrointestinal hormones after laparoscopic gastric plication.

\begin{tabular}{lllll}
\hline Variable & Preoperative & Postoperative at 6 months & P value & Postoperative at 12 months \\
\hline Ghrelin level & $551.7 \pm 17.1$ & $441.5 \pm 21.5$ & $<0.0001$ & $422.5 \pm 16.8$ \\
GLP-1 level & $33.7 \pm 2.3$ & $33.5 \pm 1.8$ & 0.76 & $33.1 \pm 1.8$ \\
\hline
\end{tabular}

\section{Discussion}

The present study aimed to investigate the impact of laparoscopic gastric plication on morbidly obese patients with type II DM with regard weight loss, changes in fasting and postprandial blood glucose levels, fasting serum insulin, and gastrointestinal hormones including ghrelin and GLP-1 at 6 and 12 months after the procedure.

The study included twenty patients, the vast majority of whom were females in line with the female predominance of obesity particularly in the developing countries [11]. All patients had type II DM and were on oral anti-diabetic medications.

LGCP proved feasible with no conversion to open surgery in any patient and with operating time ranging between 60 and 90 minutes within the range reported in the literature (50117.9 minutes). The median hospital stay was two days in line with literature that reported hospital stay after LGCP between one to 5 days [12].

Patients who underwent LGCP in the present report showed significant decrease in body weight and BMI with satisfactory loss of excess weight at 6 and 12 months. At six months the percentage of excess weight loss was $24.6 \%$, slightly lower than what has been reported by Bradnova et al. [8] (31\%). A recent systematic review [12] including 1450 patients reported the percentage of excess weight loss at six months after LGCP to range between $31.8 \%$ and $74.4 \%$.

At 12 months postoperatively the percentage of excess weight loss reached $41.4 \%$, whereas the reported range of excess weight loss at one year after LGCP was from 50 to $79 \%$ according to the several investigators [13-15]. The 
lower percentage of excess weight loss in our study may be due to non-compliance of some patients to the dietary regimen and the exercise program after surgery.

LGCP is considered a restrictive bariatric surgery similar to laparoscopic sleeve gastrectomy. Plication of the greater curvature of the stomach serves to decrease the volume capacity of the stomach, thus reduces food intake by inducing early satiety $[6,9]$. It is worthy to mention that weight loss after LSG was attributed to other factors other than volume restriction per se. Resection of the gastric fundus in sleeve gastrectomy leads to removal of most of the gastric mucosa containing ghrelin-secreting cells, thus the circulating levels of ghrelin hormone decrease in a sustained manner inducing appetite suppression [16, 17].

Although LGCP preserves the mucosa of the gastric fundus by infolding it towards the gastric lumen, it was postulated that the devascularization and infolding of the gastric fundus during LGCP may compromise perfusion of the mucosal cells and affect their endocrine function leading to decreased ghrelin secretion. The present study confirmed the hypothesis aforementioned as plasma ghrelin levels showed a significant reduction at 6 and 12 months after LGCP compared to their preoperative value. Bradnova et al [8] also demonstrated a similar decrease in ghrelin levels at 6 months after LGCP.

The improvement in DM after different bariatric procedure such as LSG and RYGB has been well demonstrated in prior studies [18, 19]. However, there is scarce literature discussing the effect of LGCP on the glycemic control in patients with type II DM. The main objective of the present study was to investigate the effect of LGCP on morbidly obese patients with type II DM.

Through the present study we observed significant improvement in type II DM in the majority of patients, this improvement was documented by remarkable improvement in the laboratory parameters as well as reduction of the dose of anti-diabetic medications required to maintain the glycemic control.

Significant reduction in the fasting and postprandial serum glucose levels and fasting serum insulin were observed denoting an improvement in the diabetic status. This observation was reported by other investigators [8] who found fasting serum insulin level declined at 6 months after LGCP, and attributed this decline to increased insulin sensitivity as assessed by HOMA-IR.

The amelioration of glucose homeostasis was further confirmed by significant decrease in $\mathrm{HbAlc}$, in concordance with other studies $[8,13]$ who reported an improvement in the preoperative diabetic status at 6 months after LGCP in $92.3 \%$ and $96.9 \%$ of patients, compared to $90 \%$ of patients in our study, as verified by a significant decrease in HbA1c levels. We also noticed further decrease in $\mathrm{HbAlc}$ levels at 12 months after LGCP, reflecting continued, sustained improvement in the glycemic control of the patients.

The improvement in type II DM observed may be related to the progressive weight loss secondary to restriction of food intake or can be attributed to the reduction in serum ghrelin levels. It has been shown that ghrelin exerts various diabetogenic effects, hence reduction of circulating levels of ghrelin may factor in amelioration of glucose homeostasis and improvement in the diabetic status after LGCP [20, 21]. Furthermore, improvement in glucose homeostasis after LGCP can be explained by changes of incretin response after LGCP in diabetic patients. The most important incretin is GIP which is secreted after meals from the $\mathrm{K}$ cells of duodenum and proximal jejunum. GIP exerts several physiologic functions including stimulation of pancreatic Beta cells to secret insulin and promoting glucose and lipid storage [22]. It has been hypothesized that beta cell resistance in type II DM diminishes the incretin effect of GIP [23].

Although the present study did not assess GIP levels before and after LGCP, other investigators did and reported a significant rise in postprandial GIP at one and six months postoperatively [8]. The remarkable increase in meal-induced GIP levels may reflect overstimulation of the $\mathrm{K}$ cells due to accelerated gastric empting owing to reduced gastric capacity after LGCP, similar to LSG, however further evidence is needed to validate this theory.

The effect of LGCP on gut hormones has been also investigated in our study, two hormones were selected to be assessed after the procedure: ghrelin and GLP-1. A significant reduction in ghrelin levels was noticed at six and 12 months after LGCP. This reduction may contribute to both weight loss and improvement in DM after the procedure. As previously shown, the ghrelin/obestatin ratio may be involved in the control of body weight through regulation of appetite and satiety levels. Hence, the significant decrease in ghrelin levels we observed can lead to reduction in the ghrelin/obestatin ratio contributing to further weight loss by suppressing the appetite [20]. On the other hand, the postoperative decrease in the ghrelin level helps eliminate its diabetogenic effects, therefore contributing to better glycemic control [16].

GLP-1 is an enteric hormone released from the L cells which are located in ileum, contrary to the $\mathrm{K}$ cells which are mainly located in the proximal bowel. The presence of dietary carbohydrates and fat in the distal bowel stimulates the secretion of GLP-1 [22].

GLP-1 tends to delay gastric emptying and improve glucose homeostasis through its insulinotropic effect, increasing insulin sensitivity, and suppressing glucagon secretion [23]. By understanding the functions of GLP-1 it can be explain why its postprandial secretion is markedly suppressed in patients with type II DM.

The present study did not observe any significant change in GLP-1 levels at 6 and 12 months after LGCP. Bradnova et al. [8] noted a higher peak of postprandial GLP-1 at one month after LGCP, nonetheless this increase in GLP-1 levels was not sustained at 6 months of follow-up. In contrast, other reports showed sustained increase in the postprandial GLP-1 secretion after LSG on long-term follow-up [24-26]. The changes in GLP-1 levels after restrictive bariatric surgery in general is attributed to faster gastric emptying and increased secretion of cholecystokinin hormone [27], however the main 
mechanism regulating GLP-1 levels after LGCP is not well elucidated.

Finally, LGCP exhibited high safety profile with no recorded mortality or major complications in line with a systematic review of 14 studies that reported no mortality and an average major complications rate of $3.7 \%$ of patients [12]. Only minor complications in the form of postoperative vomiting in the first three weeks were recorded which can be attributed to the edema of gastric wall and the volumerestricting effect of LGCP. Early postoperative vomiting has been reported after other restrictive procedures including LSG and usually resolves spontaneously and quickly.

In summary, the present study demonstrated the efficacy of LGCP not only as a bariatric procedure but as a metabolic surgery for patient with type II DM as other authors have demonstrated [28, 29]. These findings substantiate the preliminary observations by Bradnova et al [8] who studied the short-term metabolic effect of LGCP in diabetic patients. Moreover, our study showed a sustained improvement in glucose homeostasis and further reduction in BMI at 12 months after LGCP which confirms the successful effect of the procedure on the long term.

The limitations of the present study include the small number of patients included and the lack of control group for the aim of comparison of the metabolic outcome. Furthermore, some important laboratory parameter such as insulin sensitivity level, serum C-peptide level, and serum obestatin hormone level were not evaluated. Despite the limitations aforementioned, we concur with the authors of the previous study that LGCP can be considered an effective bariatric procedure with effects that may extend beyond being a restrictive bariatric surgery to include metabolic effects on glucose homeostasis and gut hormones [8].

\section{Conclusion}

LGCP is an effective bariatric procedure that achieved satisfactory weight loss at 12 months postoperatively. In addition, LGCP proved to have a positive impact on the metabolic status of patients with type II DM with significant improvement in the glycemic control as demonstrated by lowering the dose of anti-diabetic medications as well as improvement in laboratory markers as serum glucose, insulin, and ghrelin hormone levels.

\section{Conflict of Interest}

None to be disclosed by the authors

\section{References}

[1] Tsigosa C, Hainer V, Basdevant A, Finer N, Fried M, MathusVliegen E, Micic D, Maislos M, Roman G, Schutz Y, Toplak $\mathrm{H}$ and Zahorska-Markiewicz B. Management of obesity in adults: European Clinical Practice Guidelines. Eur J Obes. 2008; 2:106-16.
[2] Wing R, Phelan S (2004) Science-based solutions to obesity: what are the roles of academia, government, industry, and health care? Proceedings of a Symposium, Boston, Massachusetts, USA, 10-11 March 2004 and Anaheim, California, USA, 2 October 2004.

[3] Rucker D, Padwal R, Li SK, Curioni C and Lau Dc. Long term pharmacotherapy for obesity and overweight: updated meta-analysis. BMJ. 2007; 335 (7631):1194-9.

[4] Dixon JB, le Roux CW, Rubino F and Zimmet P. Bariatric surgery for type 2 diabetes. Lancet. 2012; 379 (9833):2300 11. PMID: 22683132. 21.

[5] Wilkinson LH, Peloso OA. Gastric (reservoir) reduction for morbid obesity. Arch Surg. 1981; 116 (5):602-5.

[6] Skrekas G, Antiochos K and Stafyla VK. Laparoscopic gastric greater curvature plication: results and complications in a series of 135 patients. Obes Surg. 2011; 21 (11):1657-63.

[7] Brethauer SA, Harris JL, Kroh $M$ and Schauer PR. Laparoscopic gastric plication for treatment of severe obesity. Surg Obes Relat Dis. 2011; 7 (1): 15-22.

[8] Bradnova O, Kyrou I, Hainer V, Vcelak J, Halkova T, Sramkova P, Dolezalova K, Fried M, McTernan P, Kumar S, Hill M, Kunesova M, Bendlova B and Vrblkov J. Laparoscopic Greater Curvature Plication in Morbidly Obese Women with Type 2 Diabetes: Effects on Glucose Homeostasis, Postprandial Triglyceridemia and Selected Gut Hormones. Obes Surg. 2014 May;24(5):718-26. doi: 10.1007/s11695-013-1143-4.

[9] Ramos A, Galvao Neto M, Galvao M, Evangelista LF, Campos JM and Ferraz A. Laparoscopic greater curvature plication: initial results of an alternative restrictive bariatric procedure. Obes Surg. 2010; 20 (7):913-8. PMID: 20407932.

[10] Taha O. Efficacy of Laparoscopic Greater Curvature Plication for Weight Loss and Type 2 Diabetes: 1-Year Follow-Up. OBES SURG (2012) 22:1629-1632.

[11] Case A, Menendez A. Sex Differences in Obesity Rates in Poor Countries: Evidence from South Africa. Economics and human biology. 2009; 7 (3):271-282. doi:10.1016/j.ehb.2009.07.002.

[12] Ji Y1, Wang Y2, Zhu J1, Shen D1. A systematic review of gastric plication for the treatment of obesity. SurgObesRelat

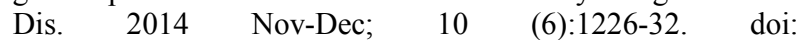
10.1016/j.soard.2013.12.003.

[13] Fried M, Dolezalova K, Buchwald JN, McGlennon TW, Sramkova P and Ribaric G. Laparoscopic greater curvature plication (LGCP) for treatment ofmorbid obesity in a series of 244 patients. Obes Surg. 2012; 22 (8):1298-307. PMID: 22648797.

[14] Mui WL, Lee DW, Lam KK, et al. Laparoscopic greater curve plication in Asia: initial experience. Obes Surg. 2013; 23 (2):179-83.

[15] Niazi M, Maleki AR, Talebpour M. Short-term outcomes of laparoscopic gastric plication in morbidly obese patients: importance of postoperative follow-up. Obes Surg. 2013; 23 (1):87-92.

[16] Scott WR, Batterham RL. Roux-en-Y gastric bypass and laparoscopicsleeve gastrectomy: understanding weight loss and improvementsin type 2 diabetes after bariatric surgery. Am J Physiol RegulIntegr Comp Physiol. 2011; 301 (1):R15-27. 
[17] Peterli R, Steinert RE, Woelnerhanssen B, et al. Metabolic andhormonal changes after laparoscopic Roux-en-Y gastric bypass and sleeve gastrectomy: a randomized, prospective trial. Obes Surg. 2012; 22 (5):740-8.

[18] Emile SH, Elfeki H, Elalfy K, Abdallah E. Laparoscopic Sleeve Gastrectomy Then and Now: An Updated Systematic Review of the Progress and Short-term Outcomes Over the Last 5 Years. Surg Laparosc Endosc Percutan Tech. 2017 Oct; 27 (5):307-317. doi: 10.1097/SLE.0000000000000418.

[19] Vidal J1, Jiménez A, de Hollanda A, Flores L, Lacy A. Metabolic Surgery in Type 2 Diabetes: Roux-en-Y Gastric Bypass or Sleeve Gastrectomy as Procedure of Choice? Curr Atheroscler Rep. 2015 Oct; 17 (10):58. doi: 10.1007/s11883015-0538-1.

[20] Broglio F, Arvat E, Benso A, et al. Ghrelin, a natural GH secretagogue produced by the stomach, induces hyperglycemia and reduces insulin secretion in humans. J Clin Endocrinol Metab. 2001; 86 (10): 5083-6.

[21] Sun Y, Asnicar M, Saha PK, et al. Ablation of ghrelin improves the diabetic but not obese phenotype of ob/ob mice. Cell Metab. 2006; 3 (5):379-86.

[22] Holst JJ. The physiology of glucagon-like peptide 1. Physiol Rev. 2007; 87 (4):1409-39.

[23] Paschetta E, Hvalryg M, Musso G. Glucose-dependent insulinotropic polypeptide: from pathophysiology to therapeutic opportunities in obesity-associated disorders. Obes Rev. 2011; 12 (10):813-28.
[24] Romero F, Nicolau J, Flores L, et al. Comparable early changes in gastrointestinal hormones after sleeve gastrectomy and RouxEn-Y gastric bypass surgery for morbidly obese type 2 diabetic subjects. SurgEndosc. 2012; 26 (8):2231-9.

[25] Dimitriadis E, Daskalakis M, Kampa M, et al. Alterations in gut hormones after laparoscopic sleeve gastrectomy: a prospective clinical and laboratory investigational study. Ann Surg. $2013 ; 257$ (4): 647-54.

[26] Papamargaritis D, le Roux CW, Sioka E, et al. Changes in gut hormone profile and glucose homeostasis after laparoscopic sleeve gastrectomy. Surg Obes Relat Dis. 2013; 9 (2):192201.

[27] Melissas J, Daskalakis M, Koukouraki S, et al. Sleeve gastrectomy - a "food limiting" operation. Obes Surg. 2008; 18 (10):1251-6.

[28] Talebpour M1, Talebpour A1, Barzin G2, Shariat Moharari R2, Khajavi MR2. Effects of laparoscopic gastric plication (LGP) in patients with type 2 diabetes, one year follow-up. J Diabetes Metab Disord. 2015 Jul 17; 14:60. doi: 10.1186/s40200-015-0188-4. eCollection 2015.

[29] Bužga M1, Maresova P2, Seidlerova A1, Zonča P1, Holéczy $\mathrm{P} 1$, Kuča K3. The influence of methods of bariatric surgery for treatment of type 2 diabetes mellitus. Ther Clin Risk Manag. 2016 Apr 15; 12:599-605. doi: 10.2147/TCRM.S96593. eCollection 2016. 